\title{
Spectral Radiative Properties of a Liquid n-Octane Droplet in the Midinfrared Region
}

\author{
Liang Zhao $\mathbb{D}^{1}{ }_{1}^{1}$ Chaoyu Jing, ${ }^{2}$ Yu Jin, ${ }^{1}$ Jiangping Chen, ${ }^{1}$ Ke Yin, ${ }^{1}$ Sujun Yang, \\ Bingqing Liu, ${ }^{1}$ Xiao Han, ${ }^{1}$ and Jianyu Tan $\mathbb{D}^{2}$ \\ ${ }^{1}$ Beijing Institute of Spacecraft System Engineering, Beijing 10094, China \\ ${ }^{2}$ School of New Energy, Harbin Institute of Technology, Weihai 264209, China \\ Correspondence should be addressed to Liang Zhao; zhao6411707@126.com and Jianyu Tan; tanjianyu@hitwh.edu.cn
}

Received 3 May 2020; Revised 2 July 2020; Accepted 9 July 2020; Published 4 August 2020

Academic Editor: Carlos Palacio

Copyright (c) 2020 Liang Zhao et al. This is an open access article distributed under the Creative Commons Attribution License, which permits unrestricted use, distribution, and reproduction in any medium, provided the original work is properly cited.

\begin{abstract}
The optical constants of a liquid hydrocarbon such as liquid n-octane are basic material properties that may be used to evaluate their thermal radiation transfer capabilities. In this study, the ellipsometry method was used to measure the optical constants of liquid n-octane in the midinfrared wavelength range of $2.0-16.0 \mu \mathrm{m}$ at temperatures of 20,50 , and $80^{\circ} \mathrm{C}$. Experimental analyses indicate the significant effect of temperature on the refractive index, although it has little effect on the absorption index. With increasing temperature, the refractive index shows a linear decrease, and reduced density leads to weaker absorption intensities. The radiative properties of n-octane droplets, including the absorption and scattering efficiency factors of single droplets with droplet radii $r=10,20,50$, and $100 \mu \mathrm{m}$ and the absorption and scattering coefficients in a droplets-air system of droplet volume fractions $f_{\mathrm{v}}=2 \%, 3 \%$, and $4 \%$, were calculated using Mie theory. The numerical results indicate that, with increasing temperature, the absorption efficiency factor slightly decreases, and the variation trend of the scattering efficiency factor is more complicated. With increasing droplet radius, the absorption efficiency factor increases within the studied wavelength range, except for certain absorption peaks, but the scattering efficiency factor tends to decrease. While the absorption is greater, the scattering is weaker for a given droplet radius. With an increasing volume fraction of n-octane droplets, the absorption and scattering coefficients increase linearly within the studied wavelength range.
\end{abstract}

\section{Introduction}

The thermal radiation transfer of liquid fuels is key to energy utilization, which plays an important role in the diagnosis and regulation of liquid-fuel ignition, liquid-droplet combustion, and fuel composition measurement and analysis in thermal devices such as automobile internal combustion engines and aerospace engines [1-10]. The optical constants (i.e., the complex refractive index $m=n-i k$, including the refractive index $n$ and the absorption index $\kappa$ ) of liquid fuels and droplet radiative properties are important material properties and the basis for calculating radiation transfer, which is indispensable in researching the optical and radiative properties of liquid fuels. The optical constants of liquid fuel, which reflect the interaction with radiation, are vital to ensure accuracy in radiative heat transfer modeling of combustion engines and diagnosis during fuel combustion [11-15].

With the development of experimental conditions, numerous experimental approaches have been proposed to investigate the optical properties of liquids, such as the attenuated total reflection (ATR) method [16-20], the transmission method [21-24], the combined transmissionreflection method $[25,26]$, and the ellipsometry method [27-33]. The ATR method is suitable for the measurement of absorption indices in strong absorption regions, whereas the transmission method is especially suitable for measuring the absorption indices of liquids in weak absorption regions. Due to the use of the Kramers-Kronig relation, the reliability of the ATR method and the transmission method largely depends on the studied spectral ranges. Moreover, the ellipsometry method directly obtains the real and imaginary 
part of the complex refractive index by measuring changes in the intensity and phase of polarized light. Due to its high sensitivity to material optical constants and high measurement accuracy, the ellipsometry method is widely used in a variety of fields. Tiwald et al. [34] measured the optical constants of several lubricants by using the infrared ellipsometry and ATR cell. In their studies, the polarized light was vertically incident on the ATR prism. They ignored the multiple reflections and transmissions among the prism surfaces. Thus, this leads to inaccuracies in the measured data. Wang et al. [35, 36] proposed a modified ellipsometry-transmission method to solve this problem, ensuring that the polarized light was vertically incident on the prism. This approach provided improved accuracy, by considering the multiple reflections and transmissions among the prism surfaces.

The importance of the absorption and scattering properties of fuel droplets in the radiative heat transfer process has been widely discussed. Absorption and scattering of thermal radiation can be applied to various problems, including heating evaporation of droplets, autoignition of fuel vapor, attenuation of radiation by droplets and sprays, and optical diagnostics of sprays [37-39]. As the absorption efficiency factor $Q_{\text {abs }}$ and scattering efficiency factor $Q_{\text {sca }}$ of droplets depend on the optical constants $(m=n-i k)$ of droplets and droplet size parameter $X=2 \pi r / \lambda$, optical constants are crucial input parameters for the calculation of radiation characteristics. Experimental studies and computer simulations of temperature and droplet size distribution in combustion equipment have been reported by various researchers $[40,41]$. However, much less attention has been paid to the temperature dependence of radiative properties of liquid-fuel droplets. Few studies have investigated the effect of droplet volume fraction on the radiative properties of the droplet-air system formed by a spray in a combustion chamber. Dombrovsky et al. [39, 42-44] calculated the radiative properties of fuel droplets in the wavelength range from 0.2 to $10 \mu \mathrm{m}$ using Mie theory. However, the optical constants used for their calculations were obtained at a single temperature, thereby neglecting the temperature dependence of optical constants. Therefore, it is necessary to study the temperature-dependent absorption and scattering efficiency factors and coefficients of fuel droplets, which are essential parameters for radiative heat transfer modeling in thermal devices.

Liquid n-octane, a component of industrial gasoline, is a colorless and transparent combustible organic compound fuel. At present, this liquid fuel is widely used in theoretical and experimental spray combustion research on the combustion chambers of internal combustion engines and aerospace engines [45-47]. It is, therefore, essential to determine the temperature-dependent optical constants and radiative properties of liquid n-octane and n-octane droplets.

In this study, the ellipsometry method is used to measure the temperature-dependent optical constants of liquid $\mathrm{n}$-octane in the spectral wavelength range $2.0-16.0 \mu \mathrm{m}$. The temperature effects on spectral optical constants are also analyzed further, and the effects of temperature and size parameters on the radiative properties of n-octane spherical droplets, including absorption and scattering efficiency factors calculated using Mie theory, are discussed. Furthermore, the effects of droplet volume fractions in a droplets-air system on absorption and scattering coefficients calculated using Mie theory are synchronously investigated.

\section{Methodology}

2.1. The Ellipsometry Method and Experimental Setup. In this study, the ellipsometry method was used to measure the optical constants of liquid n-octane using the IR-VASE ellipsometer (J.A. Woollam, Inc.). The light source of the IRVASE ellipsometer is based on the Michelson Fourier transform infrared interferometer and the wavelength range spans from 1.5 to $30 \mu \mathrm{m}$. Diagrams of ellipsometry measurements based on the "prism-liquid" system are shown in Figure 1. Zinc selenide prism glass consisting of an isosceles trapezoid with an angle of $45^{\circ}$ was used for the optical windows in the "prism-liquid" system ellipsometry apparatus. The optical constant of the zinc selenide prism was measured using the double-thickness transmission method, and the data are given in Ref. [48]. The spectroscopic ellipsometer consisted of a polarized infrared light source and detector. The specific spectral requirements of wavelength and resolution could be set directly by using the computer.

The sample cell and optical windows were used for containing the liquid to be measured. Before performing the experiment, a protective shell made of PVC was placed on the test bench and filled with inert gas. In the measurements, the polarized light was vertically incident on one side of the ATR prism. The light, reflected by the ATR prism-liquid interface, was then emitted vertically from the other side of the ATR prism. The polarized light entered the detector via the rotary compensator and analyzer to measure changes in intensity and phase. The light from the infrared light source changed its polarization state, which was expressed by the amplitude and phase, when passing through the polarizer and being received by the detector. The ellipsometric parameters, which include the amplitude difference and phase difference are denoted as $\psi$ and $\Delta$. Considering the multiple reflections among the prism-air and prism-liquid interfaces, the reflection coefficient $\widetilde{R}$ and the complex ratio $\widetilde{\rho}_{\lambda}$ at wavelength $\lambda$ are provided by $[35,36]$

$$
\begin{aligned}
\widetilde{R} & =\frac{\widetilde{t}_{01} \widetilde{r}_{12} \widetilde{t}_{10} e^{-i \beta}}{1-\widetilde{r}_{12}^{2} \widetilde{r}_{10}^{2} e^{-i 2 \beta}}, \\
\widetilde{\rho}_{\lambda} & =\tan \Psi_{\lambda} e^{i \Delta_{\lambda}}=\frac{\widetilde{R}_{p}}{\widetilde{R}_{s}}=\frac{\widetilde{r}_{12 p}}{\widetilde{r}_{12 s}}\left(\frac{1-\widetilde{r}_{12 s}^{2} \widetilde{r}_{10 s}^{2} e^{-i 2 \beta}}{1-\widetilde{r}_{12 p}^{2} \widetilde{r}_{10 p}^{2} e^{-i 2 \beta}}\right), \\
\widetilde{r}_{i j p}= & \frac{m_{j} \cos \theta_{i}-m_{i} \cos \theta_{j}}{m_{j} \cos \theta_{i}+m_{i} \cos \theta_{j}}, \\
\widetilde{r}_{i j s}= & \frac{m_{i} \cos \theta_{i}-m_{j} \cos \theta_{j}}{m_{i} \cos \theta_{i}+m_{j} \cos \theta_{j}},
\end{aligned}
$$

where the subscripts 0,1 , and 2 denote the air, prism, and liquid. The superscript " $i$ " denotes the imaginary unit. $\widetilde{R}_{p}$ 


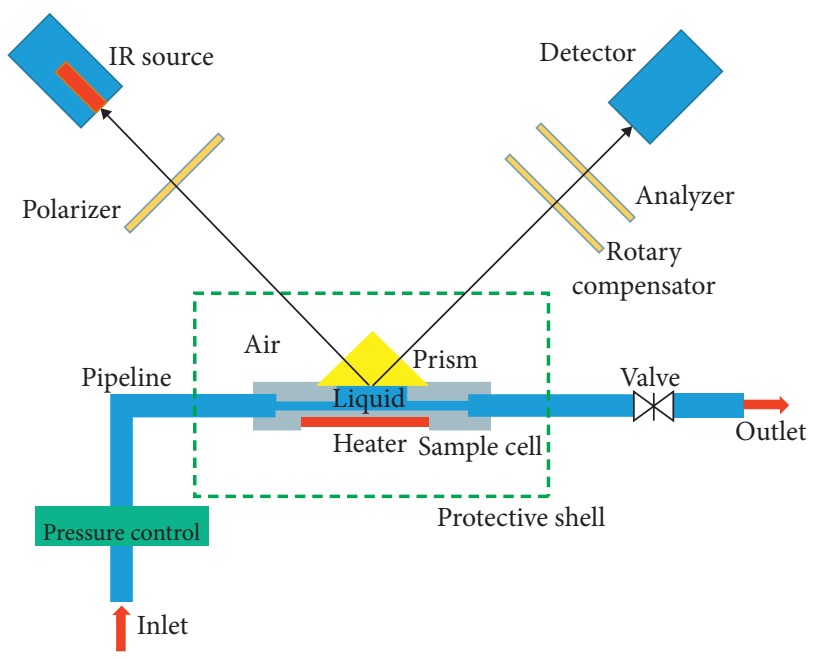

FIGURE 1: Schematic diagrams of ellipsometry measurements based on the "prism-liquid" system.

and $\widetilde{R}_{s}$ are $p$-polarized and $s$-polarized reflection coefficients. $\tilde{r}_{i j(p, s)}$ denotes the amplitude reflection coefficient at the interface between layer $i$ and $j$, which are functions of the optical constants (complex refractive index) $m_{i}=n_{i}-i k_{i}$, and $\beta=2 \pi m_{1} l / \lambda$ denotes the phase thickness, where $l$ denotes the path length of the light. $\theta_{i}$ and $\theta_{j}$ are the angles of incidence and refraction between layers $i$ and $j$ [49]. As the polarized light was vertically incident on the prism surface, $\widetilde{r}_{10 s}^{2} e^{-i 2 \beta}$ and $\widetilde{r}_{10 p}^{2} e^{-i 2 \beta}$ in equation (2) are equivalent to each other for measured media. The $\mathrm{ZnSe}$-air system measurements may be conducted to obtain $\widetilde{r}_{10 s}^{2} e^{-i 2 \beta}$ and $\widetilde{r}_{10 p}^{2} e^{-i 2 \beta}$, and then, the optical constants $m_{2}=n_{2}-i k_{2}$ of the target liquids may be obtained by solving equation (2) for the ZnSe-liquid system [35, 36].

The measurement uncertainties are evaluated using the error propagation method provided in Ref. [50]. The specific calculation method is given in an earlier paper published by our research group [36]. Deviations of the refractive indices and absorption indices are plotted with error bars.

The ceramic heater was pasted onto the back side of the liquid cell using high-temperature resistant inorganic adhesive. The liquid was connected to a real-time pressure monitor, and the pressure was maintained at $1 \mathrm{~atm}$ by adjusting the manual feed on the liquid injector. The spectral resolution was set to $23.14 \mathrm{~cm}^{-1}$, and the wavenumber range for the experimental measurements was $625-5000 \mathrm{~cm}^{-1}$, providing a measured wavelength range of $2.0-16.0 \mu \mathrm{m}$. The purity of the analytically pure n-octane provided by Shanghai Aladdin Bio-Chem Technology Co., LTD. was $99.9 \%$. As liquid n-octane has a boiling point of approximately $125-127^{\circ} \mathrm{C}$, in order to prevent the liquid from boiling during the heating measurement, which would lead to inaccurate results, the optical constants of liquid n-octane were measured at 20,50 , and $80^{\circ} \mathrm{C}$, respectively.

2.2. Radiative Property Calculations for Liquid Droplets. In the radiative heat transfer modeling of combustion engines and diagnosis during fuel combustion, liquid fuels are presented in the form of droplets. Considering the complexity of nonspherical particle calculations and similarity of fuel droplets to pure spheres, Mie theory is generally used to calculate the radiative properties of a single spherical droplet, including the absorption, scattering, and extinction efficiency factor [51, 52].

The radiative properties of a single droplet depend on two independent parameters: the size parameter $X=2 \pi r / \lambda$ and complex refractive index $m=n-i k$, where $r$ represents the droplet radius, $\lambda$ represents the wavelength of the irradiated light, and $n$ and $\kappa$ represent the refractive index and absorption index of liquid forming the droplet, respectively [53, 54].

Based on Mie theory, the extinction efficiency factor $Q_{\text {ext }}$, scattering efficiency factor $Q_{\text {sca }}$, and absorption efficiency factor $Q_{\text {abs }}$ can be calculated as follows [55-57]:

$$
\begin{aligned}
& Q_{\text {ext }}(m, \chi)=\frac{2}{\chi^{2}} \sum_{n=1}^{\infty}(2 n+1) \operatorname{Re}\left\{a_{n}+b_{n}\right\}, \\
& Q_{\text {sca }}(m, \chi)=\frac{2}{\chi^{2}} \sum_{n=1}^{\infty}(2 n+1)\left[\left|a_{n}\right|^{2}+\left|b_{n}\right|^{2}\right], \\
& Q_{\text {abs }}(m, \chi)=Q_{\text {ext }}(m, \chi)-Q_{\text {sca }}(m, \chi),
\end{aligned}
$$

where the symbol Re denotes the real parts of plurality and $a_{n}$ and $b_{n}$ denote the Mie scattering coefficients, which are calculated by [55-57]

$$
\begin{aligned}
& a_{n}=\frac{\psi_{n}^{\prime}(m \chi) \psi_{n}(\chi)-m \psi_{n}(m \chi) \psi_{n}^{\prime}(\chi)}{\psi_{n}^{\prime}(m \chi) \xi_{n}(\chi)-m \psi_{n}(m \chi) \xi_{n}^{\prime}(\chi)}, \\
& b_{n}=\frac{m \psi_{n}^{\prime}(m \chi) \psi_{n}(\chi)-\psi_{n}(m \chi) \psi_{n}^{\prime}(\chi)}{m \psi_{n}^{\prime}(m \chi) \xi_{n}(\chi)-\psi_{n}(m \chi) \xi_{n}^{\prime}(\chi)}, \\
& \xi_{n}=\psi_{n}-i \eta_{n},
\end{aligned}
$$

where $\psi_{n}$ and $\eta_{n}$ are Ricatti-Bessel functions that satisfy the following recursive relationship [55-57]:

$$
\begin{aligned}
& \psi_{n+1}(\alpha)=\frac{2 n+1}{\alpha} \psi_{n}(\alpha)-\psi_{n-1}(\alpha), \\
& \eta_{n+1}(\alpha)=\frac{2 n+1}{\alpha} \eta_{n}(\alpha)-\eta_{n-1}(\alpha),
\end{aligned}
$$

where $\psi_{-1}(\alpha)=\cos \alpha, \psi_{0}(\alpha)=\sin \alpha ; \eta_{-1}(\alpha)=-\sin \alpha$, and $\eta_{0}(\alpha)=\cos \alpha$.

In the case of a monodisperse spray, considering that fuel droplets in combustion chambers such as diesel engines are usually present with droplet radii of $10-100 \mu \mathrm{m}[43,44]$, droplets with radii $r=10,20,50$, and $100 \mu \mathrm{m}$ are calculated. For the studied wavelength range from 2.0 to $16.0 \mu \mathrm{m}$ and droplet radii from 10 to $100 \mu \mathrm{m}$, the size parameter $\chi$ of the studied droplets is from 3.9 to 314.2. Thus, the droplets may be considered to be medium $(1<\chi<10)$ and large particles $(\chi \gg 1)$ for most circumstances.

When a liquid fuel forms a spray using a nozzle, a dispersed droplet system is produced, which is composed of many droplets. In engineering applications, calculations involving polydisperse sprays are usually simplified using a 
monodisperse approximation, with droplet radii equal to an average radius of droplets in a polydisperse spray [43, 44]. To study the radiative transfer of the abovementioned droplet system, firstly, we consider the interaction between the droplets. Brewster and Tien [58] concluded the widely used criteria of independent scattering $c / \lambda=(0.905 /(f v 1 / 2)-1)$ $x / \pi \geq 0.3$, where $c$ is the interparticle clearance. In this study, the minimum particle radius $r=10 \mu \mathrm{m}$, and the corresponding minimum size parameter $\chi=3.93$ (for $\lambda=16 \mu \mathrm{m}$ ). Based on this criterion, the independent scattering condition is met along as $f v>0.39$. Therefore, the cases $(f v=2 \%, 3 \%$ and $4 \%$ ) in this study satisfy the independent scattering condition. The scattering and absorption effects of the droplet system can, then, be considered as a superposition of individual droplets. If this droplet system contains only one kind of droplet and the size distribution of the droplet is uniform, the spectral transport extinction coefficient $k_{\text {ext }}$, scattering coefficient $k_{s c a}$, and absorption coefficient $k_{a b s}$ can be expressed as [55-57]

$$
\begin{aligned}
& k_{\text {ext }}=\frac{\pi}{4} D N_{0} Q_{\text {ext }}=1.5 Q_{\text {ext }} \frac{f_{v}}{D}, \\
& k_{\text {sca }}=\frac{\pi}{4} D N_{0} Q_{\text {sca }}=1.5 Q_{\text {sca }} \frac{f_{v}}{D}, \\
& k_{\text {abs }}=\frac{\pi}{4} D N_{0} Q_{\text {abs }}=1.5 Q_{\text {abs }} \frac{f_{v}}{D},
\end{aligned}
$$

where $D$ denotes the diameter of the particle, $D=2 r, N_{0}$ denotes the number density of droplets, and $f_{\mathrm{v}}$ denotes the volume fraction of the droplets.

\section{Results and Discussion}

3.1. Spectral Optical Constants of Liquid n-Octane at Different Temperatures. The IR-VASE ellipsometer was used to measure the ellipsometric parameters $\psi$ and $\Delta$ of the "ATR prism-liquid" system, and in combining the specific mathematical models, the temperature-dependent optical constants of liquid n-octane were obtained. Figure 2 presents the optical constants of liquid n-octane measured by the ellipsometry method at 20,50 , and $80^{\circ} \mathrm{C}$ in the spectral wavelength range $2.0-16.0 \mu \mathrm{m}$. As shown in Figure 2(a), temperature has a significant effect on the refractive index $n$, and the refractive index values are within the range 1.288-1.454 for the studied wavelength and temperatures. With increasing temperature, the refractive index shows an approximately linear decrease due to the decrease in liquid density. For instance, the refractive index $n$ of liquid $\mathrm{n}$-octane at a wavelength of $3.51 \mu \mathrm{m}$ decreases from 1.454 to 1.420 as the temperature increases from $20^{\circ} \mathrm{C}$ to $80^{\circ} \mathrm{C}$, and the changing rate is about $5.67 \times 10^{-4} /{ }^{\circ} \mathrm{C}$. The values of $n$ at $7.32 \mu \mathrm{m}$ decrease from 1.395 to 1.367 as the temperature increases from $20^{\circ} \mathrm{C}$ to $80^{\circ} \mathrm{C}$, and the changing rate is about $4.67 \times 10^{-4} /{ }^{\circ} \mathrm{C}$. By contrast, Figure 2 (b) shows the much smaller effect of temperature on the absorption index $\kappa$. The measured uncertainties of the absorption indices are larger for $13-16 \mu \mathrm{m}$ wavebands due to the weakening of the light source intensity and the enhanced absorption of the zinc selenide window, thus resulting in obvious differences of the absorption index at three different temperatures. The absorption peaks in the midinfrared spectral range are results of intermolecular interactions, intramolecular bending, and symmetric or asymmetric stretching vibrations of $\mathrm{C}-\mathrm{H}$ groups. As shown, in the studied temperature region, temperature shows little effect on the absorption peaks representing $\mathrm{C}-\mathrm{H}$ vibrations.

3.2. Effect of Temperature on Absorption and Scattering Efficiency Factors of a Single Droplet. We assume that droplets of liquid n-octane that are formed during spraying are spherical. Absorption and scattering of thermal radiation by droplets can be calculated using Mie theory [44, 59]. According to Mie theory, the absorption efficiency factor $Q_{a b s}$ and scattering efficiency factor $Q_{\text {sca }}$ depend on the droplet size parameter $X=2 \pi r / \lambda$ and the complex refractive index $m=n-i k$ of liquid n-octane. The studied spectral range $2 \sim 16 \mu \mathrm{m}$ corresponds to a maximum radiative temperature range of $180 \sim 1449 \mathrm{~K}$, which is much broader than spray combustion.

Figure 3 presents the Mie-calculated absorption and scattering efficiency factors of n-octane droplets at different temperatures, with radii $r=10,20 \mu \mathrm{m}$. The absorption efficiency factor $Q_{\mathrm{abs}}$ represents the rate of the absorption cross section and the projected surface area of the spherical droplet. The scattering efficiency factor $Q_{\text {sca }}$ represents the rate of scattering cross section and the projected surface area of the spherical droplet. As shown in Figures 3(a) and 3(c), the absorption efficiency factor $Q_{a b s}$ decreases with increasing temperature due to the slight decrease of the absorption index $\kappa$ of liquid n-octane. The changing trends of $Q_{a b s}$ with increasing wavelength are similar to that of the measured absorption index $\kappa$ of liquid n-octane, which is shown in Figure 2(b). Figure 3 indicates that stronger absorption generally leads to weaker scattering of the droplet. As shown in Figures 3(b) and 3(d), the scattering efficiency factor $Q_{\text {sca }}$ of droplets shows regular damped oscillation characteristics with variations in the size parameter $\chi$. However, the trends related to increasing temperature, which leads to a decrease of $n$ and $\kappa$, on $Q_{s c a}$ differ for different spectral ranges. According to the Mie theory, the scattering efficiency factor is closely related to the complex refractive index $(m=n-i k)$ and size parameter $X=2 \pi r / \lambda$ of the droplets. For different size droplets, due to the change of the complex refractive index and size parameter, the scattering efficiency factors show different tendency with the increase of incident wavelength. For instance, the $Q_{s c a}$ of a n-octane droplets with radius $r=10 \mu \mathrm{m}$ in the wavelength range $3.7-4.3 \mu \mathrm{m}$ and $6.3-10.9 \mu \mathrm{m}$ increase with increasing temperature, although $Q_{\text {sca }}$ decreases in the wavelength ranges $4.4-5.7 \mu \mathrm{m}$ and $11.3-16.0 \mu \mathrm{m}$.

3.3. Size Parameter Effects on Absorption and Scattering Efficiency Factors of a Single Droplet. Figure 4 presents the calculated absorption and scattering efficiency factors of n-octane droplets with different droplet radii at temperatures of $20^{\circ} \mathrm{C}$. As shown in Figure 4, within the studied wavelength range except the absorption peaks around 


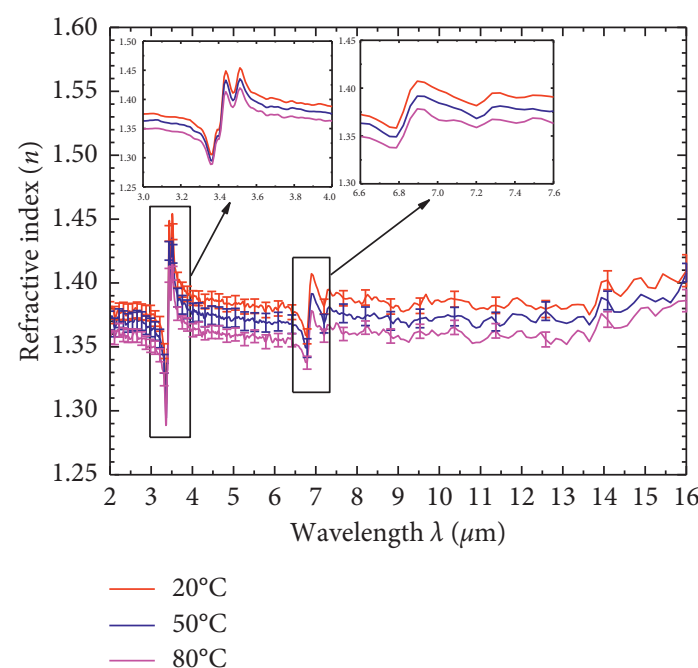

(a)

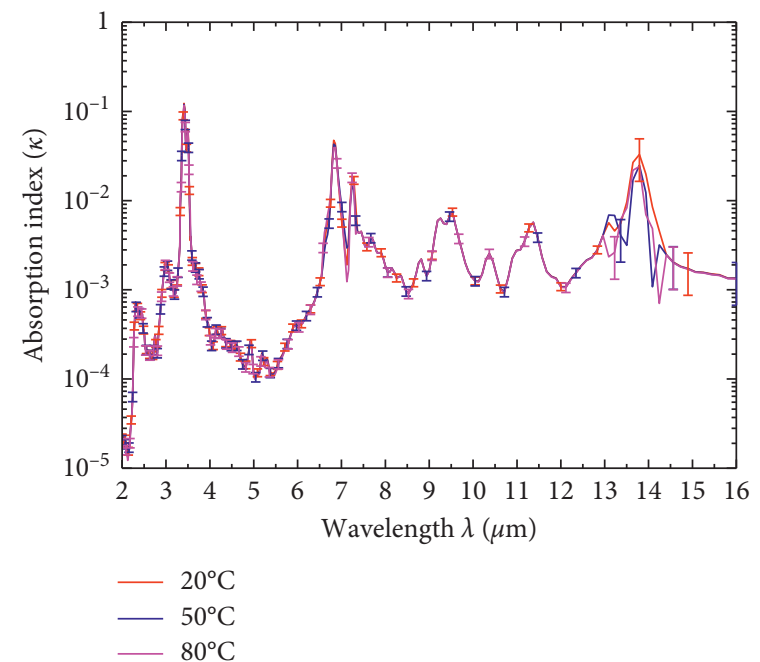

(b)

Figure 2: Refractive index $n$ and absorption index $\kappa$ of liquid n-octane at $20,50^{\circ} \mathrm{C}$, and $80^{\circ} \mathrm{C}$.
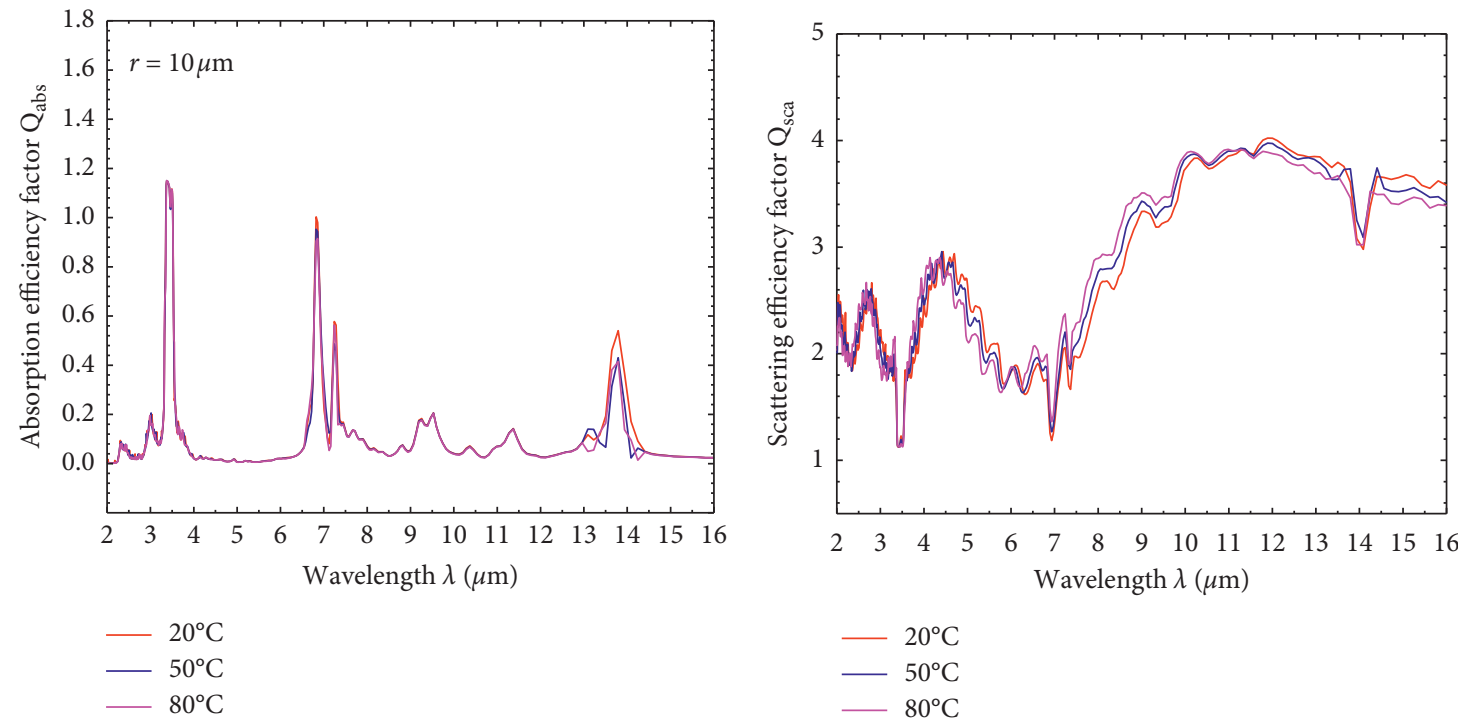

(a)

(b)

Figure 3: Continued. 


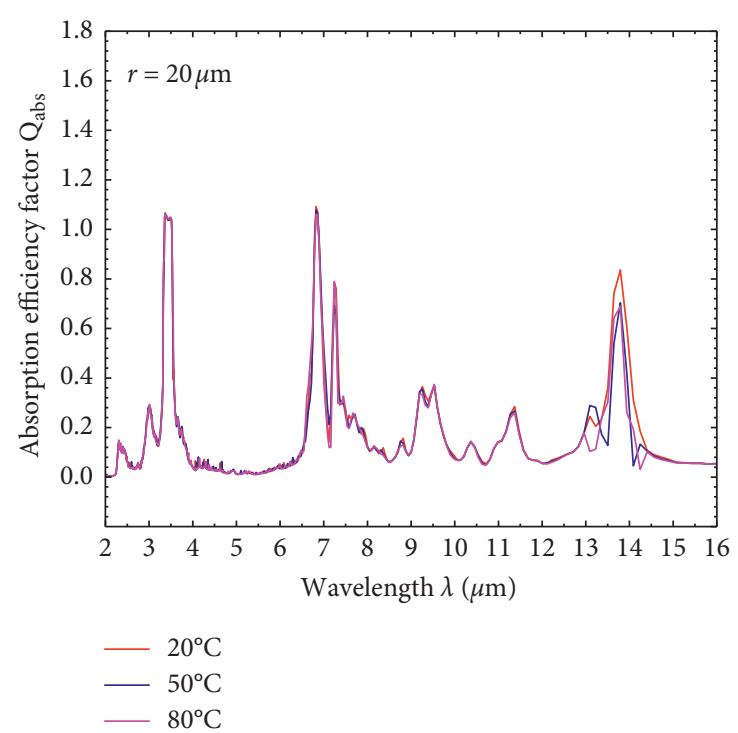

(c)

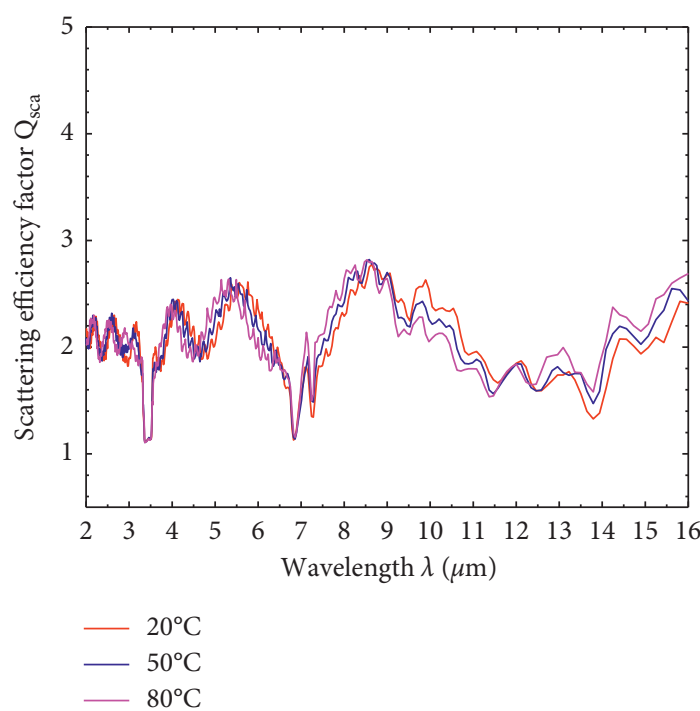

(d)

FIGURE 3: Absorption and scattering efficiency factors of a single spherical n-octane droplet with $r=10,20 \mu \mathrm{m}$.

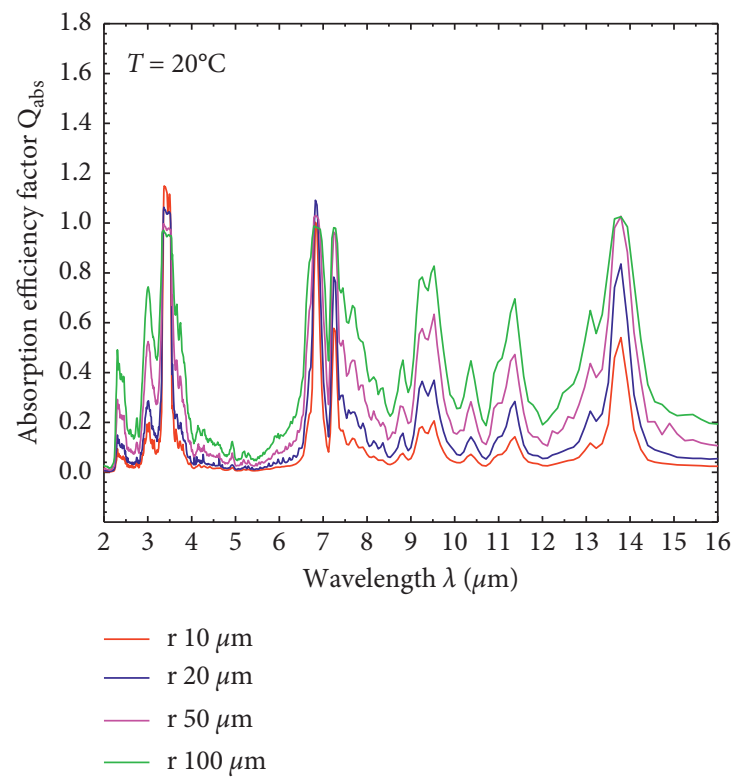

(a)

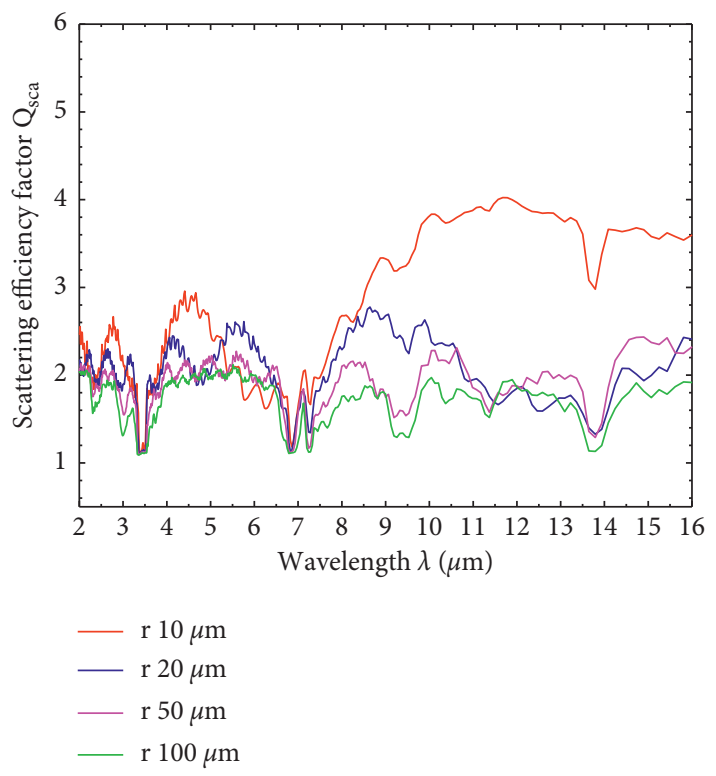

(b)

Figure 4: Absorption and scattering efficiency factors of a single spherical n-octane droplet at $20^{\circ} \mathrm{C}$.

$3.5 \mu \mathrm{m}$ and $7 \mu \mathrm{m}$, the absorption efficiency factor $Q_{\text {abs }}$ increases with increasing radius of the spherical droplet. The changing trend of the scattering efficiency factor $Q_{s c a}$ is much more complicated as the droplet radius increases. $Q_{\text {sca }}$ does not monotonically change with the increase of the droplet radius, but presents a vibrating trend around the value " 2.0 ", especially for large size droplets. With increasing droplet size parameter $\chi$, the extinction efficiency factor $\left(Q_{\text {ext }}=Q_{\text {abs }}+Q_{\text {sca }}\right)$ tends to its asymptotic value '2' for a short-wave band. It can be seen from Figure 4 that scattering is weaker where absorption is greater for a given droplet radius. With increasing droplet size parameter $\chi$, the extinction efficiency factor $\left(Q_{\text {ext }}=Q_{\text {abs }}+Q_{\text {sca }}\right)$ tends to its asymptotic value ' 2 ' for a short-wave band.

\subsection{The Absorption and Scattering Coefficients of the Droplets} System. The effects of temperature and size parameter on the absorption and scattering efficiency factors of an individual spherical droplet have been evaluated. The thermal radiation transfer properties of the droplet system in an air medium formed by a nozzle, approximated as a monodisperse system, were then, calculated using Mie theory. Figure 5 presents the Mie-calculated absorption and 


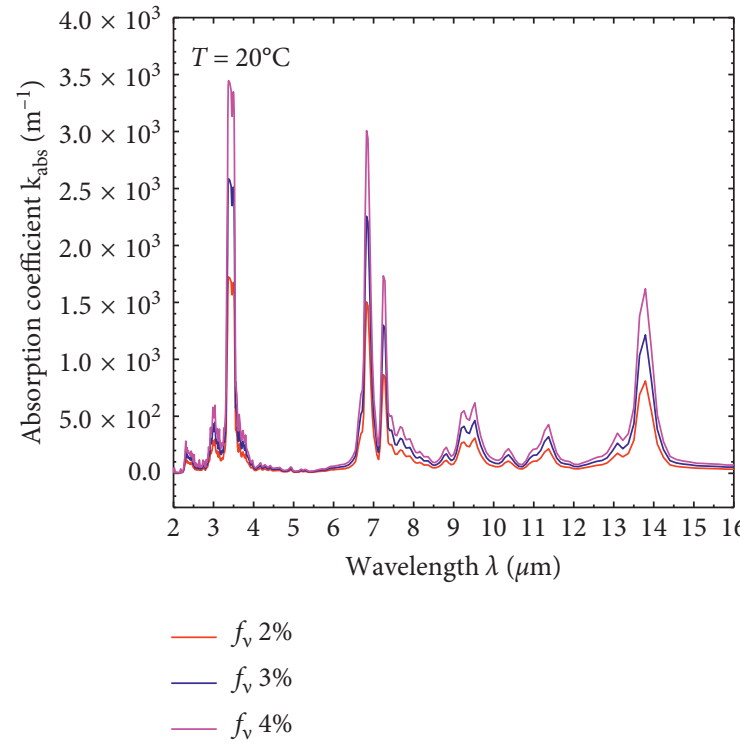

(a)

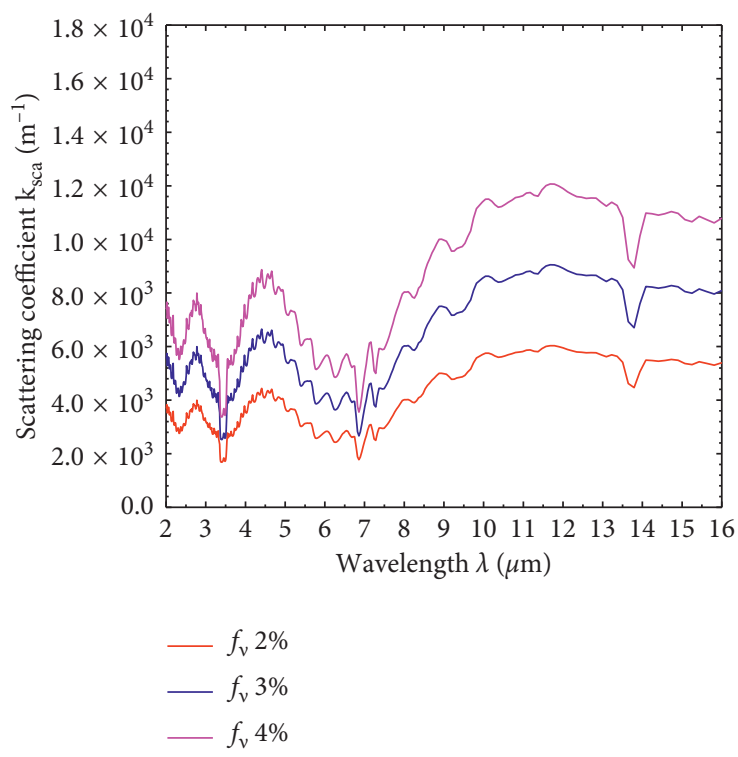

(b)

Figure 5: Absorption and scattering coefficients of the n-octane droplets-air system at $20^{\circ} \mathrm{C}$

scattering coefficients of n-octane droplets-air systems with various volume fractions at temperatures of $20^{\circ} \mathrm{C}$, with a droplet radius $r=10 \mu \mathrm{m}$. The absorption coefficient $k_{\mathrm{abs}}$ represents the rate at which incident energy is absorbed by the droplet-air system. In addition, the scattering coefficient $k_{\text {sca }}$ represents the rate at which incident energy is scattered in other directions by the system. When the temperature of the monodisperse system is equal, the radiation transfer properties of the droplet-air system are a superposition of the radiation properties of an individual droplet, as expressed in equation (14) and equation (15). Thus, as can be seen from Figure 5, the absorption and scattering coefficients of the droplet-air system increase linearly across the whole studied spectral range as the volume fraction increases.

\section{Conclusions}

In this study, the optical constants of liquid n-octane, the absorption and scattering efficiency factors of a single spherical droplet, and the absorption and scattering coefficients of droplets-air systems formed by sprays with various droplet radii and droplet volume fractions were investigated at 20,50 , and $80^{\circ} \mathrm{C}$ in the midinfrared wavelength range of $2.0-16.0 \mu \mathrm{m}$. The absorption and refractive indices of liquid n-octane were obtained experimentally using the improved ellipsometry method. The absorption and scattering efficiency factors of n-octane droplets of various temperatures and radii, together with the absorption and scattering coefficients of droplet-air systems of various volume fractions, were then calculated using Mie theory. The following conclusions were drawn.

The refractive index of liquid n-octane presents an approximately linear relationship with temperature, while temperature has a much smaller effect on the absorption index. With increasing temperature, a reduction in density leads to a smaller refractive index and weaker absorption intensities of the n-octane, which leads to the decrease of the absorption efficiency factor of a single spherical n-octane droplet. According to the Mie theory, the scattering efficiency factor is closely related to the complex refractive index and size parameter of the droplets. For different size droplets, due to the change of the complex refractive index and size parameter, the scattering efficiency factors show different tendency with the increase of incident wavelength. With increasing droplet radius, the absorption efficiency factor increases within the studied wavelength range except the absorption peaks around $3.5 \mu \mathrm{m}$ and $7 \mu \mathrm{m}$. The changing trend of the scattering efficiency factor is much more complicated as the droplet radius increases. With the increase in droplet size parameter $X$, the extinction efficiency factor $\left(Q_{\text {ext }}=Q_{a b s}+Q_{\text {sca }}\right)$ oscillates around a value of 2 for a short-wave band.

\section{Data Availability}

All data used to support the findings of this study are included within the article.

\section{Conflicts of Interest}

The authors have no conflicts of interest relevant to this manuscript.

\section{Acknowledgments}

This work was supported by the National Natural Science Foundation of China (NSFC) (51576052). 


\section{References}

[1] T. Yoshikawa and R. D. Reitz, "Effect of radiation on diesel engine combustion and heat transfer," Journal of Thermal Science and Technology, vol. 4, no. 1, pp. 86-97, 2009.

[2] T. Yao, H. Yang, L Guo et al., "The deterioration mechanism of diester aero lubricating oil at high temperature," Journal of Spectroscopy, vol. 2017, Article ID 5392864, 8 pages, 2017.

[3] M. Keyvani, M. Afrand, D. Toghraie, and M. Reiszadeh, "An experimental study on the thermal conductivity of cerium oxide/ethylene glycol nanofluid: developing a new correlation," Journal of Molecular Liquids, vol. 266, pp. 211-217, 2018.

[4] B. Karbasifar, M. Akbari, and D. Toghraie, "Mixed convection of water-aluminum oxide nanofluid in an inclined lid-driven cavity containing a hot elliptical centric cylinder," International Journal of Heat and Mass Transfer, vol. 116, pp. 1237-1249, 2018.

[5] A. Akhgar and D. Toghraie, "An experimental study on the stability and thermal conductivity of water-ethylene glycol/ TiO2-MWCNTs hybrid nanofluid: developing a new correlation," Powder Technology, vol. 338, pp. 806-818, 2018.

[6] P. Barnoon and D. Toghraie, "Numerical investigation of laminar flow and heat transfer of non-newtonian nanofluid within a porous medium," Powder Technology, vol. 325, pp. 78-91, 2018.

[7] M. Miansari, M. A. Valipour, H. Arasteh, and D. Toghraie, "Energy and exergy analysis and optimization of helically grooved shell and tube heat exchangers by using taguchi experimental design," Journal of Thermal Analysis and Calorimetry, vol. 139, no. 5, pp. 3151-3164, 2020.

[8] L. K. Foong, N. Shirani, D. Toghraie, M. Zarringhalam, and M. Afrand, "Numerical simulation of blood flow inside an artery under applying constant heat flux using newtonian and non-newtonian approaches for biomedical engineering," Computer Methods and Programs in Biomedicine, vol. 190, Article ID 105375, 2020.

[9] H. Arasteh, R. Mashayekhi, M. Ghaneifar, D. Toghraie, and M. Afrand, "Heat transfer enhancement in a counter-flow sinusoidal parallel-plate heat exchanger partially filled with porous media using metal foam in the channels' divergent sections," Journal of Thermal Analysis and Calorimetry, 2019.

[10] A. Javadzadegan, M. Joshaghani, A. Moshfegh, O. A. Akbari, H. H. Afrouzi, and D. Toghraie, "Accurate meso-scale simulation of mixed convective heat transfer in a porous media for a vented square with hot elliptic obstacle: an LBM approach," Physica A: Statistical Mechanics and Its Applications, vol. 537, Article ID 122439, 2020.

[11] D. Li, H. Qi, and G. Wu, "Determined optical constants of liquid hydrocarbon fuel by a novel transmittance method," Optik, vol. 126, no. 7-8, pp. 834-837, 2015.

[12] C. S. Cheung, C. W. Leung, and T. P. Leung, "Modelling spatial radiative heat flux distribution in a direct injection diesel engine," Proceedings of the Institution of Mechanical Engineers, Part A: Journal of Power and Energy, vol. 208, no. 4, pp. 275-283, 1994.

[13] A. Tuntomo, C. L. Tien, and S. H. Park, "Optical constants of liquid hydrocarbon fuels," Combustion Science and Technology, vol. 84, no. 1, pp. 133-140, 1992.

[14] J. Guo and Z. Liu, "Chapter 9 - heat transfer during oxy-fuel combustion and boiler design," in Oxy-Fuel Combustion, C. Zheng and Z. Liu, Eds., pp. 189-208, Academic Press, Cambridge, MA, USA, 2018.
[15] L. Xu, X.-S. Bai, M. Jia, Y. Qian, X. Qiao, and X. Lu, "Experimental and modeling study of liquid fuel injection and combustion in diesel engines with a common rail injection system," Applied Energy, vol. 230, pp. 287-304, 2018.

[16] M. Safar, D. Bertrand, P. Robert, M. F. Devaux, and C. Genot, "Characterization of edible oils, butters and margarines by fourier transform infrared spectroscopy with attenuated total reflectance," Journal of the American Oil Chemists' Society, vol. 71, no. 4, pp. 371-377, 1994.

[17] C. D. Keefe and J. E. Pickup, "Infrared optical constants, dielectric constants, molar polarizabilities, transition moments, dipole moment derivatives and raman spectrum of liquid cyclohexane," Spectrochimica Acta Part A: Molecular and Biomolecular Spectroscopy, vol. 72, no. 5, pp. 947-953, 2009.

[18] M. Tasumi and A. Sakamoto, Introduction to Experimental Infrared Spectroscopy Fundamentals and Practical Methods, John Wiley \& Sons, Chichester, England, UK, 2015.

[19] B. Grégoire, B. Dazas, F. Hubert et al., "Orientation measurements of clay minerals by polarized attenuated total reflection infrared spectroscopy," Journal of Colloid and Interface Science, vol. 567, pp. 274-284, 2020.

[20] X. He, J. Wang, F. Niu et al., "Characterization of heroin and its additives by attenuated total reflection (ATR) - fourier transform infrared spectroscopy (FTIR) and multivariate analysis," Analytical Letters, pp. 1-15, 2020.

[21] C. D. Keefe and E. A. L. Gillis, "Temperature dependence of the optical properties of liquid benzene in the infrared between 25 and $50^{\circ} \mathrm{C}$," Spectrochimica Acta Part A: Molecular and Biomolecular Spectroscopy, vol. 70, no. 3, pp. 500-509, 2008.

[22] P. Rhine, D. Williams, G. M. Hale, and M. R. Querry, "Infrared optical constants of aqueous solutions of electrolytes. Alkali halides," The Journal of Physical Chemistry, vol. 78, no. 3, pp. 238-246, 1974.

[23] T. P. Otanicar, P. E. Phelan, and J. S. Golden, "Optical properties of liquids for direct absorption solar thermal energy systems," Solar Energy, vol. 83, no. 7, pp. 969-977, 2009.

[24] X. C. Li, J. M. Zhao, L. H. Liu, and J. Y. Tan, "Optical properties of edible oils within spectral range from 300 to $2500 \mathrm{~nm}$ determined by double optical pathlength transmission method," Applied Optics, vol. 54, no. 13, pp. 38863893, 2015.

[25] C. W. Robertson and D. Williams, "Optical constants of liquid ammonia in the infrared*." Journal of the Optical Society of America, vol. 63, no. 2, pp. 188-193, 1973.

[26] T. C. Paulick, "Inversion of normal-incidence (R, T) measurements to obtain n + Ik for thin films," Applied Optics, vol. 25, no. 4, pp. 562-564, 1986.

[27] V. Tkachenko, A. Marino, and G. Abbate, "Studying nematic liquid crystals by spectroscopic ellipsometry," Journal of the Society for Information Display, vol. 18, no. 11, pp. 896-903, 2010.

[28] C. S.-C. Yang, B. R. Williamsb, M. S. Huletb, T. E. Tiwaldc, R. W. Miles, and A. C. Samuels, "Optical constants of neat liquid-chemical warfare agents and related materials measured by infrared spectroscopic ellipsometry," Proceedings of SPIE, vol. 8018, Article ID 80180M, 2011.

[29] L. Wang, C. Zhao, M. H. G. Duits, F. Mugele, and I. Siretanu, "Detection of ion adsorption at solid-liquid interfaces using internal reflection ellipsometry," Sensors and Actuators B: Chemical, vol. 210, pp. 649-655, 2015.

[30] J.-C. Lai, Y.-Y. Zhang, Z.-H. Li, H.-J. Jiang, and A.-Z. He, "Complex refractive index measurement of biological tissues 
by attenuated total reflection ellipsometry," Applied Optics, vol. 49, no. 16, pp. 3235-3238, 2010.

[31] A. J. Santos, B. Lacroix, E. Blanco et al., "Simultaneous optical and electrical characterization of $\mathrm{GaN}$ nanowire arrays by means of vis-IR spectroscopic ellipsometry," The Journal of Physical Chemistry C, vol. 124, no. 2, pp. 1535-1543, 2020.

[32] K. N. Ahmadova, "Spectroscopic ellipsometric investigation of optical parameters of oil-water thin multiple systems," International Journal of Modern Physics B, vol. 34, no. 08, Article ID 2050058, 2020.

[33] N. I. Staskov, A. A. Mohammedmuradov, N. A. Krekoten, and S. O. Parashkov, "Optical characteristics of parallel-sided quartz plates at brewster angles determined by spectral ellipsometry," Journal of Applied Spectroscopy, vol. 87, no. 1, pp. 105-111, 2020.

[34] T. E. Tiwald, D. W. Thompson, J. A. Woollam, and S. V. Pepper, "Determination of the mid-IR optical constants of water and lubricants using IR ellipsometry combined with an ATR cell," Thin Solid Films, vol. 313-314, pp. 718-721, 1998.

[35] C. C. Wang, J. Y. Tan, Y. Q. Ma, and L. H. Liu, "Ab initio molecular dynamics study of temperature and pressure-dependent infrared dielectric functions of liquid methanol," AIP Advances, vol. 7, no. 3, Article ID 035115, 2017.

[36] C. C. Wang, J. Y. Tan, Y. Q. Ma, and L. H. Liu, "Infrared optical constants of liquid palm oil and palm oil biodiesel determined by the combined ellipsometry-transmission method," Applied Optics, vol. 56, no. 18, pp. 5156-5163, 2017.

[37] R. Viskanta and M. P. Mengüç, "Radiation heat transfer in combustion systems," Progress in Energy and Combustion Science, vol. 13, no. 2, pp. 97-160, 1987.

[38] S. S. Sazhin, G. Feng, M. R. Heikal, I. Goldfarb, V. Gol'dshtein, and G. Kuzmenko, "Thermal ignition analysis of a monodisperse spray with radiation," Combustion and Flame, vol. 124, no. 4, pp. 684-701, 2001.

[39] L. A. Dombrovsky, S. S. Sazhin, E. M. Sazhina et al., "Heating and evaporation of semi-transparent diesel fuel droplets in the presence of thermal radiation," Fuel, vol. 80, no. 11, pp. 1535-1544, 2001.

[40] J. Won, S. W. Baek, and H. Kim, "Autoignition and combustion behavior of emulsion droplet under elevated temperature and pressure conditions," Energy, vol. 163, pp. 800-810, 2018.

[41] B. Wang, A. Kronenburg, G. L. Tufano, and O. T. Stein, "Fully resolved DNS of droplet array combustion in turbulent convective flows and modelling for mixing fields in interdroplet space," Combustion and Flame, vol. 189, pp. 347-366, 2018.

[42] L. Dombrovsky and S. Sazhin, "Absorption of thermal radiation in a semi-transparent spherical droplet: a simplified model," International Journal of Heat and Fluid Flow, vol. 24, no. 6, pp. 919-927, 2003.

[43] L. A. Dombrovsky, "Spectral model of absorption and scattering of thermal radiation by diesel fuel droplets," High Temperature, vol. 40, no. 2, pp. 242-248, 2002.

[44] L. Dombrovsky, S. Sazhin, S. Mikhalovsky, R. Wood, and M. R. Heikal, "Spectral properties of diesel fuel droplets," Fuel, vol. 82 , no. 1, pp. 15-22, 2003.

[45] H. Xu, M. Xiao, and W. Jianxin, "Optical diagnostics for soot measurement in the cylinder of diesel engine," Vehicle Engine, vol. 03, pp. 8-13, 2007.

[46] F. Brandi and L. A. Gizzi, "Optical diagnostics for density measurement in high-quality laser-plasma electron accelerators," High Power Laser Science and Engineering, vol. 7, p. e26, 2019.

[47] C. Binder, H. Feuk, and M. Richter, "Phosphor thermometry for in-cylinder surface temperature measurements in diesel engines," Journal of Luminescence, vol. 226, p. 117415, 2020.

[48] X. C. Li, C. C. Wang, J. M. Zhao, and L. H. Liu, "Temperaturedependent optical constants of highly transparent solids determined by the combined double optical pathlength transmission-ellipsometry method," Applied Optics, vol. 57, no. 5, pp. 1260-1266, 2018.

[49] R. M. A. Azzam and N. M. Bashara, Ellipsometry and Polarized Light, North-Holland Publishing Company, New York, NY, USA, 1987.

[50] I. Hughes and T. P. A. Hase, Measurements and Their Uncertainties: A Practical Guide to Modern Error Analysis, Oxford University. Press, New York, NY, USA, 2010.

[51] M. P. Mengüç, S. Manickavasagam, and D. A. D’Sa, “Determination of radiative properties of pulverized coal particles from experiments," Fuel, vol. 73, no. 4, pp. 613-625, 1994.

[52] O. V. Kalashnikova and I. N. Sokolik, "Modeling the radiative properties of nonspherical soil-derived mineral aerosols," Journal of Quantitative Spectroscopy and Radiative Transfer, vol. 87, no. 2, pp. 137-166, 2004.

[53] M. F. Modest, Radiative Heat Transfer, Academic Press, New York, NY, USA, 3nd edition, 2013.

[54] M. I. Mishchenko, L. D. Travis, and A. A. Lacis, Multiple Scattering of Light by Particles, Cambridge University Press, New York, NY, USA, 2006.

[55] C. F. Bohren and D. R. Huffman, Absorption and Scattering of Light by Small Particles, John Wiley \& Sons, New York, NY, USA, 2008

[56] J. Tan, Y. Xie, F. Wang, L. Jing, and L. Ma, "Investigation of optical properties and radiative transfer of $\mathrm{TiO} 2$ nanofluids with the consideration of scattering effects," International Journal of Heat and Mass Transfer, vol. 115, pp. 1103-1112, 2017.

[57] M. Q. Brewster, Thermal Radiative Transfer and Properties, John Wiley \& Sons, New York, NY, USA, 1992.

[58] M. Q. Brewster and C. L. Tien, "Radiative transfer in packed fluidized beds: dependent versus independent scattering," Journal of Heat Transfer, vol. 104, no. 4, pp. 573-579, 1982.

[59] A. L. Dombrovsky, Radiation Heat Transfer in Disperse Systems, Begell House, New York, NY, USA, 1996. 